\title{
TO THE THEORY OF MAPPINGS WITH FINITE AREA DISTORTION
}

\author{
D. Kovtonyuk and V. Ryazanov
}

November 17, 2004 (helsinki.tex)

\begin{abstract}
In all dimensions $k=1, \ldots, n-1$, we show that mappings $f$ in $\mathbb{R}^{n}$ with finite distortion of hyperarea satisfy certain modulus inequalities in terms of inner and outer dilatation of the mappings.
\end{abstract}

\section{Introduction}

Quasiconformal and quasiregular mappings have been recently generalized to several directions, see e.g. [AIKM], [GI], [HK], [IKO $\left.{ }_{1}\right],\left[\mathrm{IKO}_{2}\right],[\mathrm{IM}],[\mathrm{IR}],[\mathrm{IS}]$, $\left[\mathrm{KKM}_{1}\right],\left[\mathrm{KKM}_{2}\right],[\mathrm{KO}],\left[\mathrm{MRSY}_{1}\right],\left[\mathrm{MRSY}_{2}\right],\left[\mathrm{MV}_{1}\right],\left[\mathrm{MV}_{2}\right],\left[\mathrm{RSY}_{1}\right]-\left[\mathrm{RSY}_{3}\right]$. In all those generalizations the modulus techniques play a key role. The following concept was proposed in $\left[\mathrm{MRSY}_{1}\right]$. Let $D$ be a domain in $\mathbb{R}^{n}, n \geq 2$, and let $Q: D \rightarrow[1, \infty]$ be a measurable function. A homeomorphism $f: D \rightarrow \overline{\mathbb{R}^{n}}=$ $\mathbb{R}^{n} \cup\{\infty\}$ is called a $\mathbf{Q}$-homeomorphism if

$$
M(f \Gamma) \leq \int_{D} Q(x) \cdot \rho^{n}(x) d m(x)
$$

for every family $\Gamma$ of paths in $D$ and every admissible function $\rho$ for $\Gamma$.

Recall that, given a family of paths $\Gamma$ in $\mathbb{R}^{n}$, a Borel function $\rho: \mathbb{R}^{n} \rightarrow[0, \infty]$ is called admissible for $\Gamma$, abbr. $\rho \in \operatorname{adm} \Gamma$, if

$$
\int_{\gamma} \rho d s \geq 1
$$

for each $\gamma \in \Gamma$. The (conformal) modulus of $\Gamma$ is the quantity

$$
M(\Gamma)=\inf _{\rho \in a d m \Gamma} \int_{D} \rho^{n}(x) d m(x)
$$

with the measure and the integral by Lebesgue.

In the work $\left[\mathrm{MRSY}_{2}\right]$, the concept has been extended to mappings with branching. Note that the modulus inequality (1.1) in the definition of a $Q$-homeomorphism has first appeared for $n=2$ in connection with the so-called $B M O$-quasiconformal mappings, see $\left[\mathrm{RSY}_{1}\right]-\left[\mathrm{RSY}_{3}\right]$, cf. also $V(6.6)$ in $[\mathrm{LV}]$ in the theory of quasiconformal mappings. In this paper, we consider the modulus of 
families of surfaces of various dimensions in $\mathbb{R}^{n}$ and introduce the notation of $(k, Q)$-mappings.

Below we assume that $\Omega$ is an open set in $\mathbb{R}^{n}, n \geq 2$, and that all mappings $f$ : $\Omega \rightarrow \mathbb{R}^{n}$ are continuous. Similarly $\left[\mathrm{MRSY}_{2}\right]$, given a pair $Q(x, y)=\left(Q_{1}(x), Q_{2}(y)\right)$ of measurable functions $Q_{1}: \Omega \rightarrow[1, \infty]$ and $Q_{2}: \Omega_{*} \rightarrow[1, \infty]$ and $k=1, \ldots, n-1$, we say that a mapping $f: \Omega \rightarrow \mathbb{R}^{n}, f(\Omega)=\Omega_{*}$, is a $(\mathbf{k}, \mathbf{Q})$-mapping if

$$
M(f \Gamma) \leq \int_{\Omega} Q_{1}(x) \cdot \rho^{n}(x) d m(x)
$$

and

$$
M(\Gamma) \leq \int_{\Omega_{*}} Q_{2}(y) \cdot \rho_{*}^{n}(y) d m(y)
$$

for every family $\Gamma$ of $k$-dimensional surfaces $S$ in $\Omega$ and all $\rho \in \operatorname{adm} \Gamma$ and $\rho_{*} \in \operatorname{adm} f \Gamma$.

Given a mapping $\varphi: E \rightarrow \mathbb{R}^{n}$ and a point $x \in E \subseteq \mathbb{R}^{n}$, let

$$
L(x, \varphi)=\limsup _{y \rightarrow x} \frac{|\varphi(y)-\varphi(x)|}{|y-x|},
$$

and

$$
l(x, \varphi)=\liminf _{y \rightarrow x} \frac{|\varphi(y)-\varphi(x)|}{|y-x|} .
$$

A mapping $f: \Omega \rightarrow \mathbb{R}^{n}$ is said to be of finite metric distortion, abbr. $f \in$ $F M D$, if $f$ has $(N)$-property and

$$
0<l(x, f) \leq L(x, f)<\infty \quad \text { a.e. }
$$

Note that a mapping $f: \Omega \rightarrow \mathbb{R}^{n}$ is of $F M D$ if and only if $f$ is differentiable a.e. and has $(N)$ - and $\left(N^{-1}\right)$-properties, see Corollary 3.4 in $\left[\mathrm{MRSY}_{2}\right]$. Recall that a mapping $f: X \rightarrow Y$ between measurable spaces $(X, \Sigma, \mu)$ and $\left(X^{\prime}, \Sigma^{\prime}, \mu^{\prime}\right)$ is said to have $(\mathbf{N})$ - property if $\mu^{\prime}(f(E))=0$ whenever $\mu(E)=0$. Similarly, $f$ has the $\left(\mathbf{N}^{-1}\right)$-property if $\mu(E)=0$ whenever $\mu^{\prime}(f(E))=0$.

We say that a mapping $f: \Omega \rightarrow \mathbb{R}^{n}$ has $\left(\mathbf{A}_{\mathbf{k}}\right)$ - property if the two conditions hold:

$\left(A_{k}^{(1)}\right)$ : for a.e. $k$-dimensional surface $S$ in $\Omega$ the restriction $\left.f\right|_{S}$ has $(N)$-property;

$\left(A_{k}^{(2)}\right)$ : for a.e. $k$-dimensional surface $S_{*}$ in $\Omega_{*}=f(\Omega)$ the restriction $\left.f\right|_{S}$ has $\left(N^{-1}\right)$-property for each lifting $S$ of $S_{*}$.

Here a surface $S$ in $\Omega$ is a lifting of a surface $S_{*}$ in $\mathbb{R}^{n}$ under a mapping $f$ : $\Omega \rightarrow \mathbb{R}^{n}$ if $S_{*}=f \circ S$. We also say that a mapping $f: \Omega \rightarrow \mathbb{R}^{n}$ is of finite distortion of area in dimension $k=1, \ldots, n-1$, abbr. $f \in F A D_{k}$, if $f \in F M D$ and has the $\left(A_{k}\right)$-property. Note that analogues of $\left(A_{k}\right)$-properties and the classes $F A D_{k}$ have been first formulated in the mentioned work [MRSY ${ }_{2}$ for $k=1$ where it is additionally requested local rectifiability of $S_{*}$ and $S$ in $\left(A_{k}^{(1)}\right)-$ 
and $\left(A_{k}^{(2)}\right)$-properties, respectively. Thus, the mapping class FLD (finite length distortion) in $\left[\mathrm{MRSY}_{2}\right]$ is a subclass of $F A D_{1}$. Finally, we say that a mapping $f: \Omega \rightarrow \mathbb{R}^{n}$ is of finite area distortion, abbr. $f \in F A D$, if $f \in F A D_{k}$ for every $k=1, \ldots, n-1$.

We show that every mapping $f$ with finite area distortion is a $(k, Q)$-mapping for every $k=1, \ldots, n-1$ with

$$
Q(x, y)=\left(K_{I}(x), \sum_{z \in f^{-1}(y)} K_{O}(z)\right)
$$

where

$$
\begin{gathered}
K_{I}(x)=K_{I}(x, f)=\left\{\begin{array}{rr}
\frac{|J(x, f)|}{l\left(f^{\prime}(x)\right)^{n},} & \text { if } J(x, f) \neq 0 \\
1, & \text { if } f^{\prime}(x)=0
\end{array}\right. \\
K_{O}(x)=K_{O}(x, f)=\left\{\begin{array}{rr}
\frac{\left\|f^{\prime}(x)\right\|^{n}}{|J(x, f)|}, & \text { if } J(x, f) \neq 0 \\
1, & \text { if } f^{\prime}(x)=0
\end{array}\right.
\end{gathered}
$$

and $K_{I}(x, f)=\infty=K_{O}(x, f)$ otherwise. As usual, here $f^{\prime}(x)$ denotes the Jacobian matrix of $f$ at the point of differentiability $x, J(x, f)=\operatorname{det} f^{\prime}(x)$ is its determinant and

$$
l\left(f^{\prime}(x)\right)=\min \left\{\left|f^{\prime}(x) h\right|: h \in \mathbb{R}^{n},|h|=1\right\}
$$

and

$$
\left\|f^{\prime}(x)\right\|=\max \left\{\left|f^{\prime}(x) h\right|: h \in \mathbb{R}^{n},|h|=1\right\} .
$$

The quantity $K_{I}(x, f)$ is called the inner dilatation and $K_{O}(x, f)$ the outer dilatation of the mapping $f$.

\section{Preliminaries}

Below $H^{k}, k=1, \ldots, n-1$ denotes the $\mathbf{k}$-dimensional Hausdorff measure in $\mathbb{R}^{n}, n \geq 2$. More precisely, if $A$ is a set in $\mathbb{R}^{n}$, then

$$
\begin{gathered}
H^{k}(A)=\sup _{\varepsilon>0} H_{\varepsilon}^{k}(A), \\
H_{\varepsilon}^{k}(A)=V_{k} \inf \sum_{i=1}^{\infty}\left(\frac{\delta_{i}}{2}\right)^{k}
\end{gathered}
$$

where the infimum is taken over all countable collections of numbers $\delta_{i} \in(0, \varepsilon)$ such that some sets $A_{i}$ in $\mathbb{R}^{n}$ with diameters $\delta_{i}$ cover $A$. Here $V_{k}$ denotes the volume of the unit ball in $\mathbb{R}^{k}$. $H^{k}$ is an outer measure in the sense of Caratheodory, i.e.,

1) $H^{k}(X) \leq H^{k}(Y)$ whenever $X \subseteq Y$;

2) $H^{k}\left(\Sigma X_{i}\right) \leq \Sigma H^{k}\left(X_{i}\right)$ for each sequence $X_{i}$ of sets;

3) $H^{k}(X \cup Y)=H^{k}(X)+H^{k}(Y)$ whenever $\operatorname{dist}(X, Y)>0$. 
A set $E \subset \mathbb{R}^{n}$ is called measurable with respect to $H^{k}$ if $H^{k}(X)=H^{k}(X \cap$ $E)+H^{k}(X \backslash E)$ for every set $X \subset \mathbb{R}^{n}$. It is well-known that every Borel set is measurable with respect to any outer measure in the sense of Caratheodory, see e.g. [Sa], p. 52. Moreover, $H^{k}$ is Borel regular, i.e., for every set $X \subset \mathbb{R}^{n}$ there is a Borel set $B \subset \mathbb{R}^{n}$ such that $X \subset B$ and $H^{k}(X)=H^{k}(B)$, see e.g. [Sa], p. 53, and 2.10.1 in [Fe]. The latter implies that, for every measurable set $E \subset \mathbb{R}^{n}$, there exist Borel sets $B_{*}$ and $B^{*} \subset \mathbb{R}^{n}$ such that $B_{*} \subset E \subset B^{*}$ and $H^{k}\left(B^{*} \backslash B_{*}\right)=0$, see e.g. 2.2.3 in [Fe]. In particular, $H^{k}\left(B^{*}\right)=H^{k}(E)=H^{k}\left(B_{*}\right)$.

Let $\omega$ be an open set in $\mathbb{R}^{k}, k=1, \ldots, n-1$. A (continuous) mapping $S: \omega \rightarrow \mathbb{R}^{n}$ is called a $k$-dimensional surface $S$ in $\mathbb{R}^{n}$. Sometimes we call the image $S(\omega) \subseteq \mathbb{R}^{n}$ by the surface $S$, too. The number of preimages

$$
N(S, y)=N(S, y, \omega)=\operatorname{card} S^{-1}(y)=\operatorname{card}\{x \in \omega: S(x)=y\}
$$

is said to be a multiplicity function of the surface $S$ at a point $y \in \mathbb{R}^{n}$. In the other words, $N(S, y)$ means the multiplicity of covering of the point $y$ by the surface $S$. It is known that multiplicity function is lower semi-continuous, i.e.,

$$
N(S, y) \geq \liminf _{m \rightarrow \infty} N\left(S, y_{m}\right)
$$

for every sequence $y_{m} \in \mathbb{R}^{n}, m=1,2, \ldots$ such that $y_{m} \rightarrow y \in \mathbb{R}^{n}$ as $m \rightarrow \infty$, see e.g. [RR], p. 160. Thus, the function $N(S, y)$ is Borel measurable and hence measurable with respect to every Hausdorff measure $H^{k}$, see e.g. [Sa], p. 52 .

The $k$-dimensional Hausdorff area in $\mathbb{R}^{n}$ (or simply area) associated with a surface $S: \omega \rightarrow \mathbb{R}^{n}$ is given by

$$
S(B)=\int_{B} N(S, y) d H^{k} y
$$

for every Borel set $B$ and, more generally, for an arbitrary set which is measurable with respect to $H^{k}$ in $\mathbb{R}^{n}$. The surface $S$ is rectifiable if $S\left(\mathbb{R}^{n}\right)<\infty$.

If $\rho: \mathbb{R}^{n} \rightarrow[0, \infty]$ is a Borel function, then its integral over $S$ is defined by the equality

$$
\int_{S} \rho d S=\int_{\mathbb{R}^{n}} \rho(y) N(S, y) d H^{k} y .
$$

Given a family $\Gamma$ of $k$-dimensional surfaces $S$, a Borel function $\rho: \mathbb{R}^{n} \rightarrow[0, \infty]$ is called admissible for $\Gamma$, abbr. $\rho \in \operatorname{adm} \Gamma$, if

$$
\int_{S} \rho^{k} d S \geq 1
$$

for every $S \in \Gamma$. Given $p \in(0, \infty)$, the $\mathbf{p}-$ modulus of $\Gamma$ is the quantity

$$
M_{p}(\Gamma)=\inf _{\rho \in a d m \Gamma} \int_{\mathbb{R}^{n}} \rho^{p}(x) d m(x) .
$$

The modulus is itself an outer measure on the set of families of surfaces. 
We say that $\Gamma_{2}$ is minorized by $\Gamma_{1}$ and write $\Gamma_{2}>\Gamma_{1}$ if every $S \subset \Gamma_{2}$ has a subsurface which belongs to $\Gamma_{1}$. It is known that $M_{p}\left(\Gamma_{1}\right) \geq M_{p}\left(\Gamma_{2}\right)$, see $[\mathrm{Fu}]$, p. 176-178. We also say that a property $P$ holds for $\mathbf{p}-\mathbf{a . e .}$ (almost every) $k$-dimensional surface $S$ in a family $\Gamma$ if a subfamily of all surfaces of $\Gamma$ for which $P$ fails has the $p$-modulus zero. If $0<q<p$, then $P$ also holds for $q$-a.e. $S$, see Theorem 3 in $[\mathrm{Fu}]$. In the case $p=n$, we write simply a.e.

2.8. Remark. The definition of the modulus immediately implies that, for every $p \in(0, \infty)$ and $k=1, \ldots, n-1$

1) $p$-a.e. $k$-dimensional surface in $\mathbb{R}^{n}$ is rectifiable;

2) given a Borel set $B$ in $\mathbb{R}^{n}$ of (Lebesgue) measure zero,

$$
S(B)=0
$$

for $p$-a.e. $k$-dimensional surface $S$ in $\mathbb{R}^{n}$.

2.10. Lemma. Let $k=1, \ldots, n-1, p \in[k, \infty)$ and let $C$ be an open cube in $\mathbb{R}^{n}, n \geq 2$, whose edges are parallel to coordinate axes. If a property $P$ holds for $p$-a.e. $k$-dimensional surface $S$ in $C$, then $P$ also holds for a.e. $k$-dimensional plane in $C$ which is parallel to a $k$-dimensional coordinate plane $H$.

The latter a.e. is related to the Lebesgue measure in the corresponding $(n-k)$ dimensional coordinate plane $H^{\perp}$ which is perpendicular to $H$.

Proof. Let us assume that the conclusion is not true. Then by regularity of the Lebesgue measure $m_{n-k}$ in $H^{\perp}$ there is a Borel set $B$ such that $m_{n-k}(B)>0$ and $P$ fails for a.e. $k$-dimensional plane $S$ in $C$ which is parallel to $H$ and intersects $B$. If a Borel function $\rho: \mathbb{R}^{n} \rightarrow[0, \infty]$ is admissible for the given family $\Gamma$ of surfaces $S$ such that $\rho \equiv 0$ outside of $C_{0} \times B$ where $C_{0}$ is the projection of $C$ on $H$, then by the Hölder inequality

$$
\int_{C_{0} \times B} \rho^{k}(x) d m(x) \leq\left(\int_{C_{0} \times B} \rho^{p}(x) d m(x)\right)^{\frac{k}{p}}\left(\int_{C_{0} \times B} d m(x)\right)^{\frac{p-k}{p}}
$$

and hence by the Fubini theorem

$$
\int_{\mathbb{R}^{n}} \rho^{p}(x) d m(x) \geq \frac{\left(\int_{C_{0} \times B} \rho^{k}(x) d m(x)\right)^{\frac{p}{k}}}{\left(\int_{C_{0} \times B} d m(x)\right)^{\frac{p-k}{k}}} \geq \frac{\left(m_{n-k}(B)\right)^{\frac{p}{k}}}{\left(h^{k} \cdot m_{n-k}(B)\right)^{\frac{p-k}{k}}},
$$

i.e.,

$$
M_{p}(\Gamma) \geq \frac{m_{n-k}(B)}{h^{p-k}}
$$

where $h$ is the length of the edge of the cube $C$. Thus, $M_{p}(\Gamma)>0$ that contradicts the hypothesis of the lemma. 
The following statement is an analogue of the Fubini theorem, cf. e.g. [Sa], p. 77. It extends Theorem 33.1 in [Va], cf. also Theorem 3 in [Fu] and Lemma 2.13 in $\left[\mathrm{MRSY}_{2}\right]$.

2.11. Theorem. Let $k=1, \ldots, n-1, p \in[k, \infty)$ and let $E$ be a subset in open set $\Omega \subset \mathbb{R}^{n}, n \geq 2$. Then $E$ is measurable by Lebesgue in $\mathbb{R}^{n}$ if and only if $E$ is measurable with respect to area on $p$-a.e. $k$-dimensional surface $S$ in $\Omega$. Moreover, $|E|=0$ if and only if

$$
S(E)=0
$$

on $p$-a.e. $k$-dimensional surface $S$ in $\Omega$.

Proof. By the Lindelöf property in $\mathbb{R}^{n}$ and the minorant property of $M_{p}$, we may assume without loss of generality that $\Omega$ is an open cube $C$ in $\mathbb{R}^{n}$ whose edges are parallel to the coordinate axes.

Suppose first that $E$ is Lebesgue measurable in $\mathbb{R}^{n}$. Then by the regularity of the Lebesgue measure there exist Borel sets $B_{*}$ and $B^{*}$ in $\mathbb{R}^{n}$ such that $B_{*} \subset E \subset$ $B^{*}$ and $\left|B^{*} \backslash B_{*}\right|=0$. Thus, by 2) in Remark $2.8 S\left(B^{*} \backslash B_{*}\right)=0$ and hence $E$ is measurable by area on $p$-a.e. $k$-dimensional surface $S$ in $C$. Conversely, if the latter is true, then $E$ is measurable by area on a.e. $k$-dimensional plane $H$ in $C$ which is parallel to a $k$-dimensional coordinate plane, see Lemma 2.10. Thus, $E$ is measurable by the Fubini theorem.

Now, suppose that $|E|=0$. Then there is a Borel set $B$ such that $|B|=0$ and $E \subset B$. Then by 2) in Remark 2.8 the relation (2.12) holds for $p$-a.e. $k$-dimensional surface $S$ in $C$. Conversely, if the latter is true, then, in particular, $S(E)=0$ on a.e. $k$-dimensional plane $H$ in $C$ which is parallel to a $k$-dimensional coordinate plane, see Lemma 2.10. Thus, $|E|=0$ again by the Fubini theorem.

2.13. Remark. Say by the Lusin theorem, see e.g. 2.3.5 in [Fe], for every measurable function $\rho: \mathbb{R}^{n} \rightarrow[0, \infty]$, there is a Borel function $\rho^{*}: \mathbb{R}^{n} \rightarrow[0, \infty]$ such that $\rho^{*}=\rho$ a.e. in $\mathbb{R}^{n}$. Thus, by Theorem $2.11 \rho$ is measurable on $p$-a.e. $k$-dimensional surface $S$ in $\mathbb{R}^{n}$ for every $p \in(0, \infty)$ and $k=1, \ldots, n-1$.

A Lebesgue measurable function $\rho: \mathbb{R}^{n} \rightarrow[0, \infty]$ is said to be $\mathbf{p}$-extensively admissible for a family $\Gamma$ of $k$-dimensional surfaces $S$ in $\mathbb{R}^{n}$, abbr. $\rho \in \operatorname{ext}_{p} a d m \Gamma$, if

$$
\int_{S} \rho^{k} d S \geq 1
$$

for $p$-a.e. $S \in \Gamma$. The $\mathbf{p}$-extensive modulus $\bar{M}_{p}(\Gamma)$ of $\Gamma$ is the quantity

$$
\bar{M}_{p}(\Gamma)=\inf \int_{\mathbb{R}^{n}} \rho^{p}(x) d m(x)
$$

where the infimum is taken over all $\rho \in \operatorname{ext}_{p} a d m \Gamma$. In the case $p=n$, we use notations $\bar{M}(\Gamma)$ and $\rho \in$ ext $\operatorname{adm} \Gamma$, respectively. 
2.16. Corollary. For every $p \in(0, \infty), k=1, \ldots, n-1$, and every family $\Gamma$ of $k$-dimensional surfaces in $\mathbb{R}^{n}$,

$$
\bar{M}_{p}(\Gamma)=M_{p}(\Gamma)
$$

Indeed, $\bar{M}_{p}(\Gamma) \leq M_{p}(\Gamma)$ by definition and $\bar{M}_{p}(\Gamma) \geq M_{p}(\Gamma)$ by Remark 2.13.

\section{Modulus inequalities}

The following lemma makes possible to extend the so-called $K_{0}$-inequality from the theory of quasiregular mappings to $F A D$ mappings, see e.g. [MRV], p. 16, [Ri], p. 31, [Vu], p. 130, cf. also [KO] and [MRSY 2$]$.

3.1. Lemma. Let a mapping $f: \Omega \rightarrow \mathbb{R}^{n}$ be of finite metric distortion with $\left(A_{k}^{(1)}-\right)$ property for some $k=1, \ldots, n-1$ and let a set $E \subset \Omega$ be measurable by Lebesgue. Then

$$
M(\Gamma) \leq \int_{f(E)} K_{I}\left(y, f^{-1}, E\right) \cdot \rho_{*}^{n}(y) d m(y)
$$

for every family $\Gamma$ of $k$-dimensional surfaces $S$ in $E$ and $\rho_{*} \in \operatorname{ext}$ adm $f \Gamma$ where

$$
K_{I}\left(y, f^{-1}, E\right)=\sum_{x \in E \cap f^{-1}(y)} K_{O}(x, f)
$$

In particular, here we have in the case $E=\Omega$

$$
K_{I}\left(y, f^{-1}, D\right)=K_{I}\left(y, f^{-1}\right):=\sum_{x \in f^{-1}(y)} K_{O}(x, f) .
$$

Proof. Let $B$ be a (Borel) set of all points $x$ in $\Omega$ where $f$ has a differential $f^{\prime}(x)$ and $J(x, f)=\operatorname{det} f^{\prime}(x) \neq 0$. Then $B_{0}=\Omega \backslash B$ has the Lebesgue measure zero in $\mathbb{R}^{n}$ because $f \in F M D$. It is known that $B$ is the union of a countable collection of Borel sets $B_{l}, l=1,2, \ldots$ such that $f_{l}=\left.f\right|_{B_{l}}$ is a homeomorphism which is bi-Lipschitz, see e.g. 3.2.2 in [Fe]. Setting $B_{1}^{*}=B_{1}, B_{2}^{*}=B_{2} \backslash B_{1}$ and

$$
B_{l}^{*}=B_{l} \backslash \bigcup_{m=1}^{l-1} B_{m}
$$

we may assume that $B_{l}$ are mutually disjoint. Note that by 2) in Remark $2.8 S\left(B_{0}\right)=0$ for a.e. $k$-dimensional surface $S$ in $\Omega$ and by $\left(A_{k}^{(1)}\right)$-property $S_{*}\left(f\left(B_{0}\right)\right)=0$ where $S_{*}=f \circ S$ also for a.e. $k$-dimensional surface $S$.

Given $\rho_{*} \in$ ext $\operatorname{adm} f \Gamma$, set

$$
\rho(x)=\left\{\begin{array}{rr}
\rho_{*}(f(x))\left\|f^{\prime}(x)\right\|, & \text { for } x \in \Omega \backslash B_{0}, \\
0, & \text { otherwise. }
\end{array}\right.
$$


We may assume without loss of generality that $\rho_{*} \equiv 0$ outside of $f(E)$. Arguing piecewise on $B_{l}$, we have by 3.2.20 and 1.7.6 in [Fe] and Theorem 2.11, see also Remark 2.13, that

$$
\int_{S} \rho^{k} d S \geq \int_{S_{*}} \rho_{*}^{k} d S \geq 1
$$

for a.e. $S \in \Gamma$, i.e., $\rho \in$ ext $a d m \Gamma$. Hence by (2.17)

$$
M(\Gamma) \leq \int_{\Omega} \rho^{n}(x) d m(x) .
$$

Now, the change of variables, see e.g. [Mu], p. 31, we obtain that

$$
\int_{f\left(B_{l} \cap E\right)} K_{O}\left(f_{l}^{-1}(y), f\right) \cdot \rho_{*}^{n}(y) d m(y)=\int_{\Omega} \rho_{l}^{n}(x) d m(x)
$$

where $\rho_{l}=\rho \cdot \chi_{B_{l}}$ and every $f_{l}=\left.f\right|_{B_{l}}, l=1,2, \ldots$ is injective by the construction.

Thus, by the Lebesgue monotone convergence theorem, see e.g. [Sa], p. 27,

$$
\int_{f(E)} K_{I}\left(y, f^{-1}, E\right) \cdot \rho_{*}^{n}(y) d m(y)=\int_{\Omega} \sum_{l=1}^{\infty} \rho_{l}^{n}(x) d m(x) \geq M(\Gamma) .
$$

The next inequality is a generalized form of the $K_{I}$-inequality which is also known as Poletskii's inequality, see [Pol], [Ri], p. 49-51, and [Vu], p. 131, cf. $\left[\mathrm{MRSY}_{2}\right]$.

3.10. Lemma. Let $f: \Omega \rightarrow \mathbb{R}^{n}$ be an FMD mapping with $\left(A_{k}^{(2)}\right)$-property for some $k=1, \ldots, n-1$. Then

$$
M(f \Gamma) \leq \int_{\Omega} K_{I}(x, f) \cdot \rho^{n}(x) d m(x)
$$

for every family $\Gamma$ of $k$-dimensional surface $S$ in $\Omega$ and $\rho \in \operatorname{ext} a d m \Gamma$.

Proof. Let $B_{l}, l=0,1,2, \ldots$, be given as above in the proof of Lemma 3.1. By the construction and $(N)$-property $\left|f\left(B_{0}\right)\right|=0$. Thus, by Theorem 2.11 $S_{*}\left(f\left(B_{0}\right)\right)=0$ for a.e. $S_{*} \in f \Gamma$ and hence by $\left(A_{k}^{(2)}\right)$-property $S\left(B_{0}\right)=0$ for a.e. $S_{*} \in f \Gamma$ where $S$ is an arbitrary lifting of $S_{*}$ under the mapping $f$, i.e., $S_{*}=f \circ S$.

Let $\rho \in \operatorname{ext} a d m \Gamma$ and

$$
\tilde{\rho}(y)=\sup _{x \in f^{-1}(y) \cap \Omega \backslash B_{0}} \rho_{*}(x)
$$

where

$$
\rho_{*}(x)=\left\{\begin{array}{rr}
\rho(x) / l\left(f^{\prime}(x)\right), & \text { for } x \in \Omega \backslash B_{0}, \\
0, & \text { otherwise. }
\end{array}\right.
$$


Note that $\tilde{\rho}=\sup \rho_{l}$ where

$$
\rho_{l}(y)=\left\{\begin{array}{rr}
\rho_{*}\left(f_{l}^{-1}(y)\right), & \text { for } y \in f\left(B_{l}\right) \\
0, & \text { otherwise }
\end{array}\right.
$$

and every $f_{l}=\left.f\right|_{B_{l}}, l=1,2, \ldots$ is injective. Thus, the function $\tilde{\rho}$ is measurable, see e.g. [Sa], p. 15 .

Arguing as in (3.6) we obtain that

$$
\int_{S_{*}} \tilde{\rho}^{k} d S_{*} \geq \int_{S} \rho^{k} d S \geq 1
$$

for a.e. $S_{*}=f \circ S \in f \Gamma$ and, thus, $\tilde{\rho} \in$ ext $a d m f \Gamma$. Hence (2.17) yields

$$
M(f \Gamma) \leq \int_{f(\Omega)} \tilde{\rho}^{n}(y) d m(y) .
$$

Further, by the change of variables we have that

$$
\int_{B_{l}} K_{I}(x, f) \cdot \rho^{n}(x) d m(x)=\int_{f(\Omega)} \rho_{l}(y) d m(y) .
$$

Finally, by Lebesgue's theorem we obtain the desired inequality

$$
\int_{\Omega} K_{I}(x, f) \cdot \rho^{n}(x) d m(x)=\sum_{l=1}^{\infty} \int_{f(\Omega)} \rho_{l}(y) d m(y)=\int_{f(\Omega)} \sum_{l=1}^{\infty} \rho_{l}(y) d m(y) \geq M(f \Gamma) .
$$

Combining Lemmas 3.1 and 3.10 we come to the main result.

3.18. Theorem. Let a mapping $f: \Omega \rightarrow \mathbb{R}^{n}$ belong to the class $F A D_{k}$ for some $k=1, \ldots, n-1$. Then $f$ is a $(k, Q)$-mapping in the dimension $k$ with

$$
Q(x, y)=\left(K_{I}(x, f), K_{I}\left(y, f^{-1}\right)\right) .
$$

3.20. Corollary. Every $F A D$ mapping $f$ is a $(k, Q)$-mapping for each $k=1, \ldots, n-1$ with $Q$ given in (3.19).

3.21. Remark. If $K_{I}(f)=\operatorname{ess} \sup K_{I}(x, f)<\infty$, then (3.11) for $k=1$ yields the Poletskii inequality:

$$
M(f \Gamma) \leq K_{I}(f) M(\Gamma)
$$

for every path family in $\Omega$. If $K_{O}(f)=\operatorname{ess} \sup K_{O}(x, f)<\infty$ and $E$ is a Borel set with $N(f, E)<\infty$, then we have from (3.2) the usual form of the $K_{O}$-inequality:

$$
M(\Gamma) \leq N(f, E) K_{O}(f) M(f \Gamma)
$$

for every path family in $E$.

Acknowledgements. The research has been partially supported by the Fund of Fundamental Investigations of Ukraine, Grant 01.07/00241. 


\section{References}

[AIKM] Astala K., Iwaniec T., Koskela P. and Martin G., Mappings of BMO-bounded distortion, Math. Annalen 317 (2000), 703-726.

[EG] Evans L.C. And Gapiery R.F., Measure Theory and Fine Properties of Functions, CRC Press, Boca Raton, 1992.

[Fe] Federer H., Geometric Measure Theory, Springer, Berlin etc., 1969.

[Fu] Fuglede B., Extremal length and functional completion, Acta Math. 98 (1957), 171-219.

[GI] Gehring F.W. And Iwaniec T., The limit of mappings with finite distortion, Ann. Acad. Sci. Fenn. Math. 24 (1999), 253-264.

[HK] Heinonen J. And Koskela P., Sobolev mappings with integrable dilatations, Arch. Rational Mech. Anal. 125 (1993), 81-97.

$\left[\mathrm{IKO}_{1}\right]$ Iwaniec T., Koskela P, and Onninen J., Mappings of finite distortion: compactness, Ann. Acad. Sci. Fenn. Math. 27, no. 2 (2002), 391-417.

$\left[\mathrm{IKO}_{2}\right]$ Iwaniec T., Koskela P, and Onninen J., Mappings of finite distortion: monotonicity and continuity, Invent. Math. 144, no. 3 (2001), 507-531.

[IM] Iwaniec T. And Martin G., Geometrical Function Theory and Non-linear Analysis, Clarendon Press, Oxford, 2001.

[IR] Ignat'ev A. And Ryazanov V., To the theory of removable singularities of space mappings, Proc. of Inst. Appl. Math. \& Mech. 8 (2003), 25-38.

[IS] Iwaniec T. And ŠverÁk V., On mappings with integrable dilatation, Proc. Amer. Math. Soc. 118 (1993), 181-188.

[KKM $\left.{ }_{1}\right]$ Kauhanen J., Koskela P. And Maly J., Mappings of finite distortion: discreteness and openness, Arch. Rational Mech. Anal. 160 (2001), 135-151.

$\left[\mathrm{KKM}_{2}\right]$ Kauhanen J., Koskela P. and Maly J., Mappings of finite distortion: condition N, Michigan Math. J. 49 (2001), 169-181.

[KO] Koskela P. and Onninen J., Mappings of finite distortion: capacity and modulus inequalities, Dept. Math. Stat., University of Jyväskylä, Preprint 257 (2002), 1-32.

[LV] Lehto O. and Virtanen K., Quasiconformal Mappings in the Plane, Springer, New York etc., 1973.

[MRV] Martio O., Rickman S., VäısäLÄ J., Definitions for quasiregular mappings, Ann. Acad. Sci. Fenn. Ser. A I. Math. 448 (1969), 1-40.

[MRSY ${ }_{1}$ ] Martio O., Ryazanov V., Srebro U. and Yakubov E., On Q-homeomorphisms (to appear)

[MRSY $\left.{ }_{2}\right]$ Martio O., Ryazanov V., Srebro U. and Yakubov E., Mappings with finite length distortion (to appear)

[Mu] Muller S., Higher integrability of determinants and weak convergence in $L^{1}$, J. Reine Angew. Math. 412 (1990), 20-34.

[MV $\mathrm{MV}_{1}$ Manfredi J.J. And Villamor E., Mappings with integrable dilatation in higher dimensions, Bull. Amer. Math. Soc. 32, no. 2 (1995), 235-240.

$\left[\mathrm{MV}_{2}\right]$ Manfredi J.J. And Villamor E., An extension of Reshetnyak's theorem, Indiana Univ. Math. J. 47, no. 3 (1998), 1131-1145.

[Pol] PoletskiI, The modulus method for non-homeomorphic quasiconformal mappings, Mat. Sb. 83 (125) (1970) 261-272. 
[Ri] Rickman S., Quasiregular Mappings, Springer, Berlin etc., 1993.

[RR] Reimann H.M. And Rychener T., Funktionen Beschrankter Mittlerer Oscillation, Springer, Berlin etc., 1975.

[RSY ${ }_{1}$ ] Ryazanov V., Srebro U. and Yakubov E., To the theory of BMO-quasiregular mappings, Dokl. Akad. Nauk Rossii 369, no 1 (1999), 13-15.

[RSY $\left.{ }_{2}\right]$ Ryazanov V., SRebro U. and Yakubov E., BMO-quasiconformal mappings, J. d'Analyse Math. 83 (2001), 1-20.

[RSY $\left.{ }_{3}\right]$ Ryazanov V., Srebro U. and Yakubov E., Plane mappings with dilatation dominated by functions of bounded mean oscillation, Sib. Adv. in Math. 11, No. 2 (2001), 94-130.

[Sa] Saks S., Theory of the Integral, New York, Dover Publ. Inc., 1964.

[Va] VÄıs̈̈LÄ J., Lectures on n-Dimensional Quasiconformal Mappings, Lecture Notes in Math. 229, Berlin etc., Springer-Verlag, 1971.

[Vu] Vuorinen M., Conformal Geometry and Quasiregular Mappings, Lecture Notes in Math. 1319, Berlin etc., Springer-Verlag, 1988.

Institute of Applied Mathematics and Mechanics, National Academy of Sciences of Ukraine 